Objectives: To assess the body composition $(\mathrm{BC})$ and to identify the frequency of sarcopenia (SP) in SSc patients.

Methods: A total of 44 women who met the ACR/EULAR 2013 classification criteria were included. Mean age was $53,2+8,8$ years. The median disease duration was $7,0[4,0 ; 12,0]$ years. $26(59,1 \%)$ patients had limited and $18(40,9 \%)$ diffuse cutaneous subtype. Body composition was measured using Dual-energy $X$-ray absorptiometry (DXA) of whole body. The appendicular lean mass index (ALMI) was calculated as the ratio of appendicular lean mass (ALM) to height $(\mathrm{kg} / \mathrm{m} 2)$. Handgrip measurement and chair stand test were performed. Physical function was measured with the Short Physical Performance Battery (SPPB). SP was diagnosed in agreement with the 2019 revised consensus on definition and diagnosis of SP of the European Working Group on Sarcopenia in Older People 2 (EWGSOP2): handgrip <16kg, chair stand test $>15$ seconds for 5 rises, ALM $<15 \mathrm{~kg}$ or ALMI $<5.5 \mathrm{~kg} / \mathrm{m} 2$. Severe SP was detected if the patient additionally had gait speed $\leq 0.8 \mathrm{~m} / \mathrm{s}$ or SPPB $\leq 8$-point score. Overfat was defined as body fat percentage $>35 \%$.

Results: The median bone mineral content was $2.0[1.8 ; 2.2] \mathrm{kg}$, total lean mass - 39,5 [35,7; 45,5] kg, ALM - 16,3 [14,5;19,4] kg, ALMI - 6,5 [5,7; 7,2] kg/m2, trunk fat mass - 13,5 $[9,1 ; 16,7] \mathrm{kg}$ and total fat mass - 26,6 [20,1;34,5] kg. Body fat percentage was $38,8 \%[34,2 ; 42,7]$

$9(20,5 \%)$ women had low ALM and low ALMI, $6(13,6 \%)$ - only low ALM. Healthy BC was found in $5(11,3 \%)$, low ALM or low ALMI - in $7(15,9 \%)$, overfat - in 24 $(54,5 \%)$, low ALM + overfat - in $8(18,2 \%)$ patients. We found no differences in BC between SSc patients with limited and diffuse cutaneous subtype.

Low muscle strength (SP probable) was found in 21 (47,7\%) women, meanwhile confirmed SP (low muscle strength and muscle mass) was diagnosed in 10 $(22,7 \%)$ patients, among them $5(11,4 \%)$ persons had severe SP. No significant difference in SP frequency among patients with limited and diffuse cutaneous SSc $4(15,4 \%)$ and $6(33,3 \%)$, respectively, $(p=0,27)$. Osteoporosis was found in $6(60 \%)$ patients with SP without differences in SSc subtypes.

Conclusion: Healthy BC was found only in $11,3 \%$ cases, while overfat - in $72,7 \%$ and low ALM - in $34,1 \%$ SSc patients. SP was detected in $22.7 \%$ of women, among them in half of cases - severe SP, without any differences between the limited and diffuse subtypes of the disease.

Disclosure of Interests: None declared

DOI: 10.1136/annrheumdis-2021-eular.970

\section{POS0840 $\quad$ RISK OF MALNUTRITION IN PATIENTS WITH SYSTEMIC SCLEROSIS-ASSOCIATED INTERSTITIAL LUNG DISEASE (SSC-ILD): FURTHER ANALYSES OF THE SENSCIS TRIAL}

E. Volkmann ${ }^{1}$, Z. Mcmahan ${ }^{2}$, S. Johnson ${ }^{3}$, V. Smith ${ }^{4}$, S. Jouneau ${ }^{5}$, C. Miede ${ }^{6}$, M. Alves ${ }^{7}$, A. Herrick ${ }^{8}$ on behalf of the SENSCIS trial investigators.. ${ }^{1}$ University of California, David Geffen School of Medicine, Department of Medicine, Division of Rheumatology, Los Angeles, United States of America; ${ }^{2}$ Johns Hopkins University School of Medicine, Division of Rheumatology, Baltimore, United States of America; ${ }^{3}$ Toronto Western and Mount Sinai Hospitals, University of Toronto, Toronto Scleroderma Program, Department of Medicine, Toronto, Canada; ${ }^{4}$ Ghent University Hospital, Department of Rheumatology and Internal Medicine, Ghent, Belgium; ${ }^{5}$ Competences Centre for Rare Pulmonary Diseases, University of Rennes, CHU Rennes, Inserm, EHESP, Irset (Institut de recherche en santé, environnement et travail) - UMR_S 1085, Department of Respiratory Medicine, F-35000 Rennes, France; ${ }^{6}$ mainanalytics $\mathrm{GmbH}$, Statistics, Sulzbach (Taunus), Germany; ${ }^{7}$ Boehringer Ingelheim International $\mathrm{GmbH}, \mathrm{TA}$ Inflammation Med, Ingelheim am Rhein, Germany; ${ }^{8}$ The University of Manchester, Salford Royal NHS Foundation Trust, Centre for Musculoskeletal Research, Manchester, United Kingdom

Background: Gastrointestinal (Gl) involvement is common in patients with SSc and may lead to weight loss and malnutrition. In the SENSCIS trial in patients with SSC-ILD, nintedanib reduced the rate of decline in forced vital capacity (FVC) (mL/year) over 52 weeks, with mainly Gl adverse events. The Malnutrition Universal Screening Tool (MUST) was developed to identify adults who are at risk of malnutrition and has been used in studies of patients with SSc.

Objectives: To evaluate nutritional status over 52 weeks in the SENSCIS trial based on a modified MUST score.

Methods: The SENSCIS trial enrolled patients with SSc-ILD with first non-Raynaud symptom $\leq 7$ years before screening, extent of fibrotic ILD $\geq 10 \%$ on HRCT and FVC $\geq 40 \%$ predicted. Patients who had taken a stable dose of mycophenolate for $\geq 6$ months were eligible to participate. Patients were randomised to receive nintedanib $150 \mathrm{mg}$ bid or placebo. Dose reductions to $100 \mathrm{mg}$ bid and treatment interruptions were allowed to manage adverse events and specific recommendations were provided for the management of diarrhoea. We calculated a modified MUST score at baseline and weeks 12, 24, 36 and 52 based on (A) BMI, (B) weight loss (week 12 vs baseline; week 24 vs week 12; week 36 vs week 24; week 52 vs week 36 ), and (C) a surrogate for acute disease effect (if the patient had any serious adverse event that led to hospitalisation between weight assessments and received $\geq 1$ medication from the WHO classification code for "solutions for parenteral nutrition" for $\geq 5$ days). MUST score at baseline was based solely on BMI. With scores ranging from 0 to 6 , the risk of malnutrition is defined as low (score $=0$ ), medium (score $=1$ ) or high (score $\geq 2$ ).

Results: Among 576 patients who received nintedanib $(n=288)$ or placebo $(n=288)$, mean (SD) age at baseline was 54.0 (12.2) years, weight was 69.7 (15.9) $\mathrm{kg}$ and BMl was $25.9(5.0) \mathrm{kg} / \mathrm{m}^{2}$; median time since onset of first non-Raynaud symptom was 3.4 years; and $75.2 \%$ of patients were female. In the nintedanib and placebo groups, respectively, MUST scores suggested that $74.0 \%$ and $78.1 \%$ of patients were at low risk of malnutrition at baseline and remained at low risk at their last measurement (Table 1). At weeks 12 and 52, respectively, mean (SD) MUST scores were $0.3(0.6)$ and $0.4(0.7)$ in the nintedanib group and $0.2(0.5)$ and $0.2(0.6)$ in the placebo group. At weeks 12, 24, 36 and 52 respectively, the proportions of patients at low risk of malnutrition were $81.8 \%$, $80.9 \%, 72.9 \%$ and $76.5 \%$ in the nintedanib group and $86.6 \%, 86.4 \%, 88.3 \%$ and $80.8 \%$ in the placebo group; the proportions at medium risk were $12.1 \%, 13.1 \%$ $18.0 \%$ and $13.5 \%$ in the nintedanib group and $8.5 \%, 8.6 \%, 5.8 \%$ and $13.1 \%$ in the placebo group; and the proportions of patients at high risk were $6.1 \%, 5.6 \%$, $8.3 \%$ and $9.6 \%$ in the nintedanib group and $4.9 \%, 4.3 \%, 4.7 \%$ and $5.4 \%$ in the placebo group.

Table 1. Risk of malnutrition at baseline and at last assessment over 52 weeks in the SENSCIS trial.

\begin{tabular}{|c|c|c|c|c|c|c|c|c|}
\hline \multirow{3}{*}{$\begin{array}{l}\text { Last } \\
\text { assessment } \\
\text { of risk }\end{array}$} & \multicolumn{4}{|c|}{ Nintedanib $(\mathrm{n}=\mathbf{2 8 8})$} & \multicolumn{4}{|c|}{ Placebo $(n=288)$} \\
\hline & \multicolumn{4}{|c|}{ Baseline risk } & \multicolumn{4}{|c|}{ Baseline risk } \\
\hline & Low & Medium & High & Total & Low & Medium & High & Total \\
\hline Low & $213(74.0)$ & $1(0.3)$ & 0 & 214 (74.3) & 225 (78.1) & $7(2.4)$ & 0 & $232(80.6)$ \\
\hline Medium & 31 (10.8) & $8(2.8)$ & 0 & 39 (13.5) & $20(6.9)$ & $14(4.9)$ & $4(1.4)$ & 38 (13.2) \\
\hline High & $13(4.5)$ & $8(2.8)$ & $7(2.4)$ & $28(9.7)$ & $3(1.0)$ & $2(0.7)$ & $10(3.5)$ & $15(5.2)$ \\
\hline Missing & $7(2.4)$ & 0 & 0 & $7(2.4)$ & $3(1.0)$ & 0 & 0 & $3(1.0)$ \\
\hline Total & 264 (91.7) & 17 (5.9) & $7(2.4)$ & $288(100)$ & 251 (87.2) & $23(8.0)$ & $14(4.9)$ & $288(100)$ \\
\hline
\end{tabular}

MUST score ranges from 0 to 6 . Score of $0=$ low risk: score of $1=$ medium risk; score of $\geq 2=$ high risk. Baseline MUST score was based solely on BMI.

Conclusion: In the SENSCIS trial, scores based on a modified MUST indicated that most patients treated with nintedanib were at low risk of malnutrition at baseline and remained at low risk over 52 weeks. The proportions of patients at high risk of malnutrition were low but were numerically greater in patients who received nintedanib than placebo. Management of disease manifestations and gastrointestinal adverse events that may be associated with weight loss is important to reduce the risk of malnutrition in patients with SSc-ILD treated with nintedanib.

Acknowledgements: The SENSCIS trial was funded by Boehringer Ingelheim. Medical writing support was provided by FleishmanHillard Fishburn, London, UK The authors meet criteria for authorship as recommended by the International Committee of Medical Journal Editors (ICMJE).

Disclosure of Interests: Elizabeth Volkmann Consultant of: Boehringer Ingelheim, Grant/research support from: Corbus and Forbius, Zsuzsanna McMahan: None declared, Sindhu Johnson Consultant of: Boehringer Ingelheim, CSL Behring and Ikaria, Grant/research support from: Bayer, Boehringer Ingelheim Corbus, GlaxoSmithKline, Merck and Roche, Vanessa Smith Speakers bureau: Boehringer Ingelheim and Janssen, Consultant of: Boehringer Ingelheim, Grant/ research support from: Belgian Fund for Scientific Research in Rheumatic diseases (FWRO), Boehringer Ingelheim, Research Foundation - Flanders (FWO) and Janssen, Stéphane Jouneau Speakers bureau: Actelion, AIRB, AstraZeneca Bristol-Myers Squibb, Boehringer Ingelheim, Chiesi, Genzyme, GlaxoSmithKline, LVL Mediphar, Mundipharma, Novartis, Pfizer, Roche and Sanofi, Consultant of: AIRB, Boehringer Ingelheim, Novartis and Roche, Grant/research support from: AIRB, Boehringer Ingelheim, LVL Mediphar, Novartis and Roche, Corinna Miede Employee of: Currently an employee of mainanalytics $\mathrm{GmbH}$, contracted by Boehringer Ingelheim, Margarida Alves Employee of: Currently an employee of Boehringer Ingelheim, Ariane Herrick Speakers bureau: Janssen, Consultant of: Boehringer Ingelheim, Camurus, CSL Behring and Gesynta Pharma, Grant/ research support from: Actelion and Gesynta Pharma DOI: 10.1136/annrheumdis-2021-eular.1083

\section{POS0841 RISK FACTORS OF LOW BONE MINERAL DENSITY IN WOMEN WITH SYSTEMIC SCLEROSIS}

A. Efremova $^{1}$, N. Toroptsova ${ }^{2}$, N. Demin ${ }^{2}$, O. Dobrovolskaya ${ }^{2}$, O. Nikitinskaya ${ }^{2}$ ${ }^{1}$ V.A. Nasonova Research Institute of Rheumatology, Department of Osteoporosis, Moscow, Russian Federation; ${ }^{2}$ V.A. Nasonova Research Institute of Rheumatology, Department of Osteoporosis, Moscow, Russian Federation 